\title{
SARS-CoV-2: Comparison of Active Immunity Acquired by Natural Exposure versus Inoculation of HCW
}

\author{
Shagun Bhatia Shah ${ }^{1}$, Rajiv Chawla ${ }^{2}$, Anurag Mehta ${ }^{3}$, Nitin Bansal ${ }^{4}$, \\ Chamound Rai Jain $^{5}$, Anuj Prakash ${ }^{6}$, Shalini Mishra ${ }^{7}$, Sudhir Kumar Rawal ${ }^{8}$ \\ ${ }^{1}$ Consultant Anaesthesiologist; Rajiv Gandhi Cancer Institute and Research Centre; Sec-5; Rohini; Delhi. \\ ${ }^{2}$ Director Anaesthesiology; Rajiv Gandhi Cancer Institute and Research Centre; \\ ${ }^{3}$ Senior Consultant and Head; Laboratory and Transfusion Services; Rajiv Gandhi Cancer Institute and Research \\ Centre; Sec-5; Rohini; Delhi. \\ ${ }^{4}$ Infectious Disease Officer; Rajiv Gandhi Cancer Institute and Research Centre; Sec-5; Rohini; Delhi. \\ ${ }^{5}$ Consultant Cardiologist; Rajiv Gandhi Cancer Institute and Research Centre; Sec-5; Rohini; Delhi. \\ ${ }^{6}$ Senior Biochemist (Consultant I) \& Quality Manager- Laboratory Services; Rajiv Gandhi Cancer Institute and \\ Research Centre; Sec-5; Rohini; Delhi. \\ ${ }^{7}$ Consultant Paediatric Surgical Oncology; Rajiv Gandhi Cancer Institute and Research Centre; Sec-5; Rohini; \\ Delhi. \\ ${ }^{8}$ Managing Director and Consultant Surgical Oncology; Rajiv Gandhi Cancer Institute and Research Centre; \\ Sector -5; Rohini; Delhi.
}

Corresponding Author: Shagun Bhatia Shah

\begin{abstract}
Background: The immune response to vaccination is not expected to be the same in COVID-19 survivors and COVID-naïve population. There is a deficiency of specific guidelines regarding vaccination (number and temporal spacing of doses) for the burgeoning subset of population which has recovered from COVID-19 due to paucity of literature.

Methodology: This prospective, observational, single-centric, case-control study compares the IgG-antibody count at two time-points (post first and second inoculation) in 33 COVID-survivors (Group-P) and 31 COVID-naïve (Group-N) health care workers. Adverse events postinoculation were recorded. Paired and independent sample t-tests were used for intra and intergroup comparison, respectively. Data was expressed as bar-charts, dotted box-whisker and dot-line plots. P-value $<0.05$ was considered statistically significant.
\end{abstract}

Results: The mean IgG count in Group-P was $23.58 \pm 2.06$ arbitrary units (AU) whereas that in Group-N was $12.20 \pm 8.01$ AU after first inoculation. All Group-P patients had a count $\geq$ 20AU. The mean IgG-count in Group-P was $25.48 \pm 2.79 \mathrm{AU}$ whereas that in Group-N was $16.40 \pm 6.55 \mathrm{AU}$ after the second dose of vaccination. $10 \mathrm{HCW}$ in Group-N but none in Group-P developed COVID-19 after second inoculation.

Conclusion: All COVID-naïve persons must mandatorily be administered both doses of vaccination (their IgG-titres reach protective levels only after the second inoculation). The second inoculation provides no additional benefit in COVID-survivors ( 2 crore 73 lac Indians) since their IgG-counts cross the $18 \mathrm{AU}$ mark (high protection), after the first dose itself. Triage of this resource to COVID-naïve population can ameliorate vaccine-shortage. Separate explicit guidelines for vaccination of corona-survivors may need formulation on a priority basis. Although both are protective, natural infection confers more robust immunity than vaccination. Pre-emptive paracetamol nullifies most post-inoculation adverse effects.

Key Words: Adverse events; COVID-naïve; COVID-survivor; Immunity; Vaccination

\section{INTRODUCTION}

Active acquired immunity may be induced by natural exposure to a pathogen or by vaccination. Covishield (ChAdOx1nCOV; manufactured by Serum Institute of 
India; developed by Oxford-AstraZeneca) Covaxin (BBV-152; developed by Bharat Biotech and Indian Council of Medical Research) and Sputnik-V (developed by Moscow-based Gamaleya Research Institute; manufactured by Serum Institute of India) are the three vaccines available in India. ${ }^{[1]}$ Although the trinity of vaccines equipped with emergency use authorization is enthusiastically being utilized in the nationwide vaccination drive, there exist no separate guidelines on vaccination of individuals who are corona-survivors. Does the above subset of 2 crore 73 lac Indians ${ }^{[2]}$ require to get vaccinated? Vaccine prioritization based on age is already being practiced in India. Immunity status should also be taken into account during this triage of population during the vaccine drive for effective utilization of this limited resource. ${ }^{[3]}$ Would vaccination lead to greater adverse reactions in individuals with a pre-existent strong humoral immunity as compared to COVID-naïve HCW? Plasma B-cells rapidly divide when exposed to a second dose of the same virus/vaccine. The process of B-cell maturation is also activated by the booster dose resulting in better targeted antibodies. ${ }^{[4]}$ Hence, a booster dose produces antibodies faster than the initial exposure/inoculation (which is known to produce an IgG antibody response only after 1 month of exposure). So, would the booster dose/second exposure produce an antibody response as early as 1week to 10 days as against the 4-weeks taken by the first exposure?

Our primary objective was to compare the post-vaccination IgG-antibody levels in COVID-survivors with postvaccination IgG-antibody levels in COVIDnaïve HCW after the first and second doses of vaccination. Our secondary objectives were to record any adverse events post vaccination and to find out whether a correlation exists between the antibody titres and severity of signs and symptoms of any reaction. We also aimed to find out if the time of onset of antibody response post vaccination was quicker in $\mathrm{HCW}$ previously exposed to SARS-CoV-2 by noting response at 7-10 days in these $\mathrm{HCW}$ and at 28 days post vaccination (on the day of the scheduled booster dose) in COVID-naïve HCW.

\section{METHODOLOGY}

This prospective observational, twoarm, single-centric case control study was carried out after obtaining written informed consent from all health care workers, approval from the scientific committee and institutional review board and CTRI registration (CTRI/2021/04/032729). 64 adult ASA I-II HCW were included in the study conducted at a premiere tertiary care oncology centre. All HCW of either sex, aged 18-70 years with a history of testing positive for SARS-CoV-2 and with moderate (4.62-18 arbitrary units; AU) or high (>18.45 AU) IgG antibody titre, were included in the study. HCW who did not get vaccinated against COVID-19 were excluded from the study. The vaccine employed was Covishield (chimpanzee adenoviral vector vaccine). The study group (Group-P; n=33) comprised HCW with a history of being COVID-positive and developing moderate/high antibody titre, while the control group (Group-N; $n=31$ ) comprised those HCW who were asymptomatic for COVID-19 and hence never required to get a Reverse Transcriptase Polymerase Chain Reaction (RT-PCR) test done or had a negative RTPCR test report. The signs and symptoms pertaining to any adverse vaccine related reaction were recorded. Antibody response post first dose of vaccination was noted at 7 10 days post vaccination (and again on the day of the scheduled booster dose if IgGtitres<4.62AU were recorded after the first test) in the study group while IgG antibody titres was recorded at 30 days post first inoculation (on the day of scheduled booster dose) in the control group. IgG-titres were also noted 1 month after the second inoculation in both the groups. VITROS Immunodiagnostic Products Anti-SARSCoV-2 IgG 2, the IgG-test kit employed for 
serological tests at RGCIRC, is based on the high throughput automated chemiluminescence immunoassay (CLIA) technology. Antibodies tested are IgG isotypes produced against the spike protein of SARS-CoV-2. It is an immunometric test utilizing ECi/ECiQ, 3600, 5600/XT 7600 system requiring an intravenous serum sample of $20 \mu \mathrm{L}$ tested at $37^{\circ} \mathrm{C}$ with incubation time $37 \mathrm{mins}$ and time to first result 48mins. 90.0\% Positive Percent Agreement to PCR and $100 \%$ clinical specificity (95\% CI: 99.1-100.0\%), are additional features. ${ }^{[5]}$

Signal for test sample divided by the Signal at Cutoff (Cutoff value) is the result expressed in arbitrary units (AU) which is referred to as the IgG-count/level or S/C value henceforth. As per the VITROS brochure, IgG-count <1AU is considered non-reactive (non-responders), IgG-values between 1-1.46AU provide minimal protection, those between 1.46-18.45AU confer medium levels of protection and values above 18.45AU provide high levels of protection. High concordance was observed between the VITROS Anti-SARSCoV-2 IgG 2 signal to cut-off value (S/C) and the neutralizing antibody titer. Although the Limit of Quantitation (LoQ) is set at 0.127 , the assay cut-off remains at $\mathrm{S} / \mathrm{C}$ of $1.00 \mathrm{AU}$. S/C values of $1,5,10,15$ and 20 $\mathrm{AU}$ correspond to neutralizing antibody titres of 1:40, 1:80, 1:160, 1:320 and 1:640 respectively. ${ }^{[5,6]}$ An $\mathrm{S} / \mathrm{C}$ of $12 \mathrm{AU}$ corresponds with $1: 250,50 \%$ inhibition of infection of cultured cells neutralization antibody titer which is the optimal value set by FDA for COVID-19 convalescent plasma donation although 1:160 and 1:80 titers are also acceptable in emergency situations. 1:320 is the optimal titer as per European Commission guidelines. ${ }^{[6]}$

\section{Sample size calculation}

Keeping Type-I/alpha-error at 0.05 and power of the study at $80 \%$, difference in means as 5, expected standard deviations as 5 and 8 respectively, $29 \mathrm{HCW}$ in each group were required. Allowing for dropouts, we enrolled $33 \mathrm{HCW}$ in the study group and $31 \mathrm{HCW}$ in the control group.

\section{Statistical Analysis}

Paired sample t-test and independent sample t-test were used for intragroup and intergroup comparison for normally distributed, continuous/quantitative variables (expressed as mean $\pm \mathrm{SD}$ ). Chi-square test was performed for categorical/qualitative variables (expressed as numbers and percentage). MannWhitney test was utilised for non-Gaussian data (expressed as median and range). Medcalc statistical software (version 15; MedCalc Software Ltd; Ostend, Belgium) was utilized to express data as bar charts, dotted box-whisker and dot-line plots. Pvalue $<0.05$ was considered statistically significant. Microsoft Excel 2010 (Microsoft Corp., Redmond, WA, USA) was utilised for the analysis of descriptive statistics.

\section{RESULTS}

The Strobe checklist ${ }^{[7]}$ for observational case-control study was adhered to. The mean age of HCW in group-P $(40.41 \pm 10.06$ years) and Group-N (40.81 \pm 10.88 years) was comparable (table$1)$. The genderwise distribution across both groups $(48.4 \%$ males in Group-P versus $54.8 \%$ males in Group-N) was also comparable $(\mathrm{p}=0.843$; Chi Squared test; Table-1)

\begin{tabular}{|c|c|c|c|c|c|c|c|}
\hline \multicolumn{8}{|c|}{ Table-1: Demographic parameters } \\
\hline & $\mathrm{n}$ & \multicolumn{2}{|c|}{ Mean } & \multicolumn{2}{|c|}{ SD } & 95\% CI & p-value \\
\hline Age (Group-P) & 34 & \multicolumn{2}{|l|}{40.41} & \multicolumn{2}{|l|}{10.06} & 36.90 to 43.92 & 0.9385 \\
\hline Age (Group-N) & 31 & \multicolumn{2}{|l|}{40.61} & \multicolumn{2}{|l|}{10.88} & 36.62 to 44.60 & (t-test) \\
\hline Sex (Group-P) & 34 & \multirow[t]{2}{*}{ Male } & $15(48.4 \%)$ & \multirow[t]{2}{*}{ Female } & $16(58.6 \%)$ & \multirow{2}{*}{\multicolumn{2}{|c|}{$\begin{array}{l}0.8430 \\
\text { (Chi-squared test) }\end{array}$}} \\
\hline Sex (Group-N) & 31 & & $17(54.8 \%)$ & & $14(45.2 \%)$ & & \\
\hline
\end{tabular}

The mean IgG count in Group-P was $23.58 \pm 2.06 \mathrm{AU}$ whereas that in Group-N was 12.20 $\pm 8.01 \mathrm{AU}$ after first dose of vaccination (Table-2). 
Table-2: Comparison of IgG counts between Group-P and Group-N at two time points

\begin{tabular}{|l|l|l|l|l|l|l|}
\hline Timing & Group & n & Mean IgG & SD & 95\% CI & p-value \\
\hline Post $1^{\text {st }}$ dose & Group-P & 33 & 23.58 & 2.06 & 22.85 to 24.31 & $<0.0001$ \\
\cline { 2 - 7 } & Group-N & 31 & 12.20 & 8.01 & 9.26 to 15.13 & (Welch test) \\
\hline \multirow{2}{*}{ Post $2^{\text {nd }}$ dose } & Group-P & 29 & 25.48 & 2.79 & 24.42 to 26.54 & $<0.0001$ \\
& Group-N & 30 & 16.40 & 6.55 & 13.96 to 18.85 & (Welch test) \\
\hline
\end{tabular}

All patients in Group-P had a count $\geq 20 \mathrm{AU}$ on rounding off the decimals, the highest and lowest values being 27AU and 19.7AU, respectively. Box-whisker plots with boxes depicting the median (middle line) and first and third quartiles and the whiskers showing 1.5 times the Inter Quartile Range above and below the box showed no outliers. All observations (IgG levels in individual $\mathrm{HCW}$ ) are plotted as dots. A downsloping connecting line is seen connecting the mean values of Group-P with Group-N (square markers), bars represent the mean value and the error bars represent 95\% confidence interval for mean (Figure$1)$.
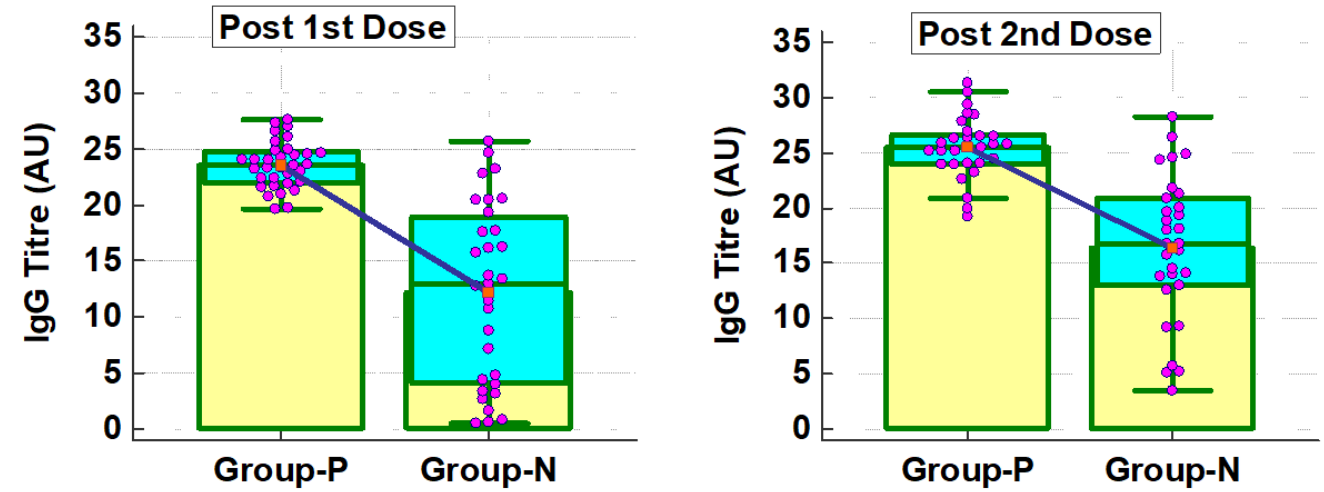

Figure-1: Dotted box-whisker plots depicting comparison of IgG counts between COVID-survivors and COVID-naïve healthcare workers at two time points

In Group-N, three $\mathrm{HCW}$ had $\mathrm{IgG}$ level <1AU, six HCW had a count $<$ 4.62AU, another fourteen $\mathrm{HCW}$ had counts between 4.62 and 18AU and remaining eight HCW had counts >18AU.

Four HCW in Group-P refrained from taking the second dose of vaccination while one HCW in Group- $\mathrm{N}$ resigned from the hospital services and did not get his antibody titres tested after the second dose of vaccination. The mean $\mathrm{IgG}$ levels in remaining 29 Group-P HCW were 25.48 2.79AU whereas that in remaining 30 Group-N HCW was $16.40 \pm 6.55$ AU after the second dose of vaccination (Table-2).

Table-2: Comparison of IgG counts between Group-P and Group-N at two time points

\begin{tabular}{|l|l|l|l|l|l|l|}
\hline Timing & Group & n & Mean IgG & SD & 95\% CI & p-value \\
\hline Post $1^{\text {st }}$ dose & Group-P & 33 & 23.58 & 2.06 & 22.85 to 24.31 & $<0.0001$ \\
\cline { 2 - 7 } & Group-N & 31 & 12.20 & 8.01 & 9.26 to 15.13 & (Welch test) \\
\hline \multirow{2}{*}{ Post $2^{\text {nd }}$ dose } & Group-P & 29 & 25.48 & 2.79 & 24.42 to 26.54 & $<0.0001$ \\
& Group-N & 30 & 16.40 & 6.55 & 13.96 to 18.85 & (Welch test) \\
\hline
\end{tabular}

All patients in Group-P had an IgGcount $\geq 19 \mathrm{AU}$, the highest and lowest values being $31.3 \mathrm{AU}$ and $19.2 \mathrm{AU}$, respectively. In Group-N, one $\mathrm{HCW}$ had an IgG-count $<4.62 \mathrm{AU}$ corresponding to mild immunity. Another $15 \mathrm{HCW}$ had IgG S/C count between 4.62 and 18AU corresponding to moderate levels of protection and remaining $14 \mathrm{HCW}$ had $\mathrm{IgG}$ count $>18$ AU corresponding to high levels of protection.

Out of these $30 \mathrm{HCW}$ in Group-N, 10 contracted COVID-19 after vaccination ( 9 more than one month after the $2^{\text {nd }}$ dose and one HCW 2 weeks after the first dose). Three of them had an IgG count $<1 \mathrm{AU}$ and another three $<5 \mathrm{AU}$ while the remaining four $\mathrm{HCW}$ had titres 13.4, 13.7, 16.2 and 
17.7AU respectively one month after their $1^{\text {st }}$ dose of vaccination.

None of the HCW in Group-P (including the $4 \mathrm{HCW}$ who did not take the second dose) contracted COVID-19 infection again to date.
The sample size, mean IgG-titres with standard deviation and $95 \%$ confidence intervals considered for intragroup paired sample t-test (Table-3; Figure-2) gave a pvalue of 0.0001 for Group-P and 0.0008 for Group-N.

Table-3: Intragroup paired sample t-test comparing IgG counts following first and second dose of vaccination for HCW within a group

\begin{tabular}{|l|l|l|l|l|l|l|}
\hline Timing & Group & n & Mean IgG & SD & 95\% CI & p-value \\
\hline Post $1^{\text {st }}$ dose & Group-P & 33 & 23.58 & 2.06 & 22.85 to 24.31 & $<0.0001$ \\
\cline { 2 - 7 } & Group-N & 31 & 12.20 & 8.01 & 9.26 to 15.13 & (Welch test) \\
\hline \multirow{2}{*}{ Post $2^{\text {nd }}$ dose } & Group-P & 29 & 25.48 & 2.79 & 24.42 to 26.54 & $<0.0001$ \\
\cline { 2 - 6 } & Group-N & 30 & 16.40 & 6.55 & 13.96 to 18.85 & (Welch test) \\
\hline
\end{tabular}
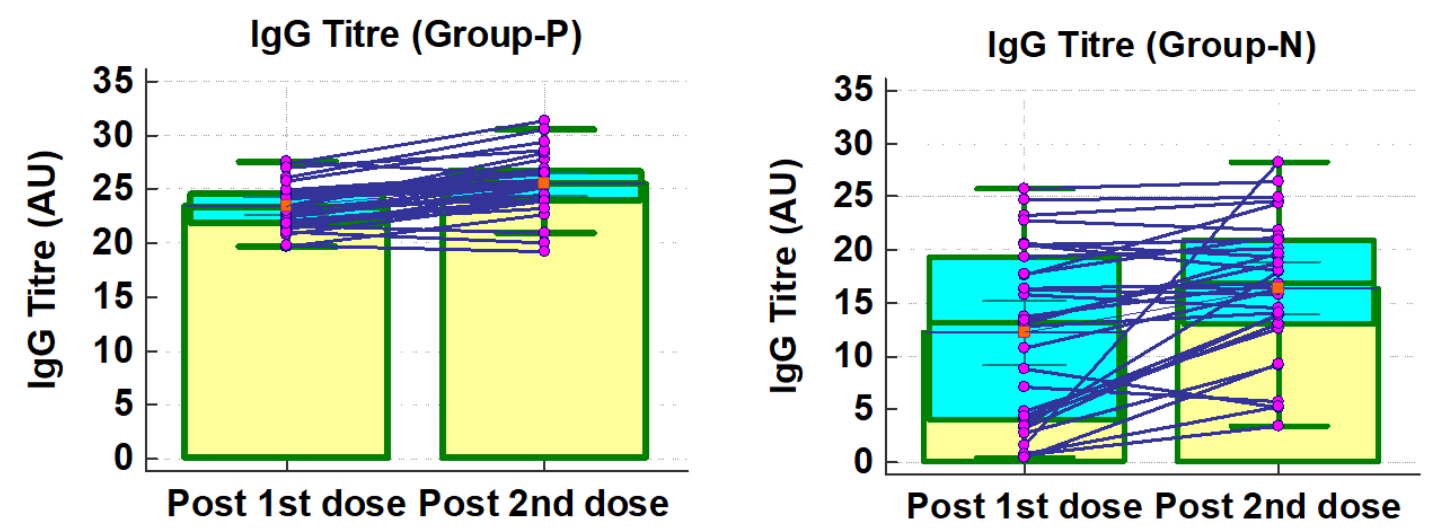

Figure-2: Trends in IgG count over time within COVID-survivors and COVID-naïve health care workers

Adverse events recorded after first dose of vaccination in Group-P include fever (9 HCW), chills (2 HCW), headache
(6 $\mathrm{HCW})$, myalgia (12 $\mathrm{HCW})$, malaise (10 $\mathrm{HCW})$ and pain at injection site $(9 \mathrm{HCW}$; Figure-3)

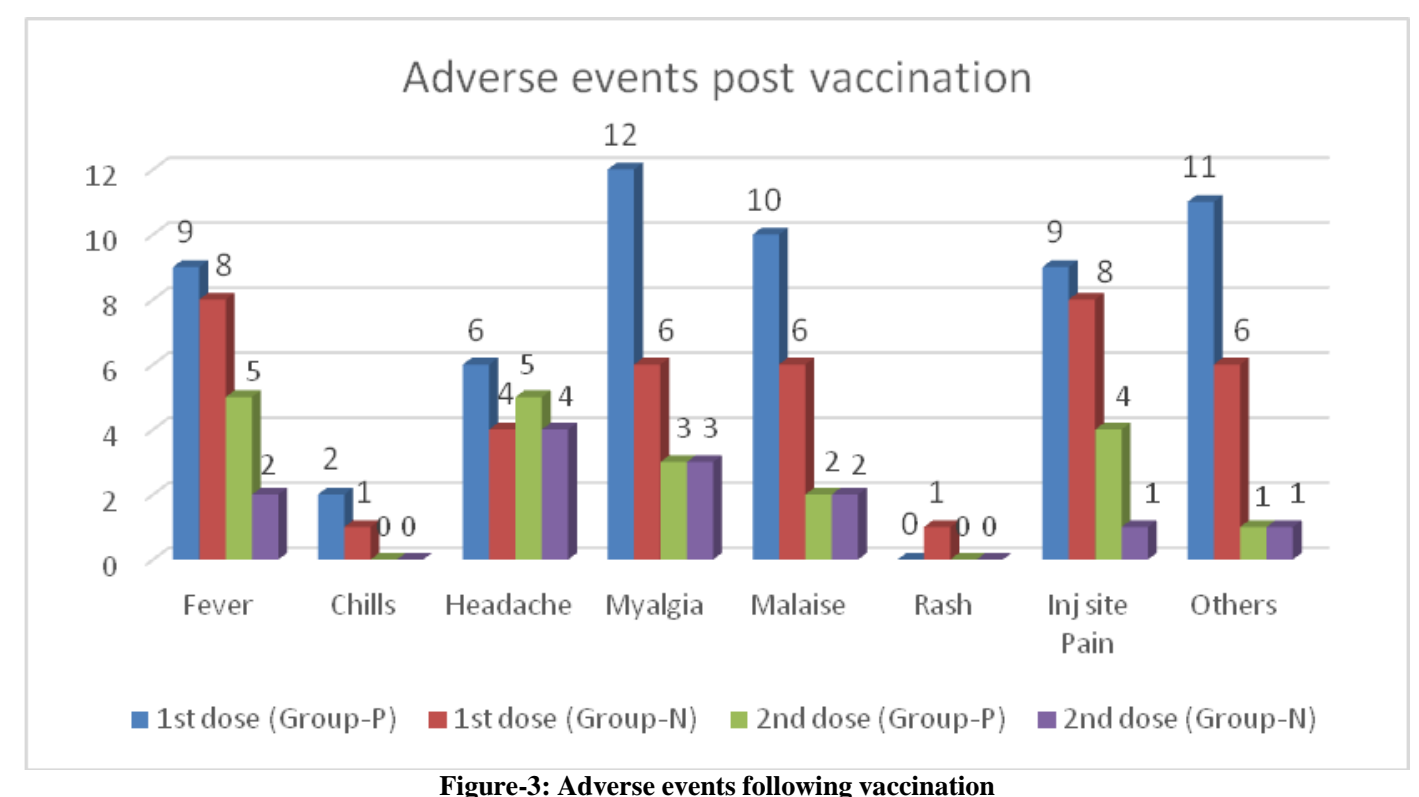

Figure-3: Adverse events following vaccination

Other complications were somnolence and sore throat in two HCW each and swelling at injection site, dizziness, joint pain, hot flushes, insomnia and arm pain in one $\mathrm{HCW}$ each. Adverse events observed in Group- $\mathrm{N}$ after the first dose of vaccination were fever $(8 \mathrm{HCW})$, chills (1 HCW), headache (4 HCW), 
myalgia (6 HCW), malaise (6 HCW) and pain at injection site $(8 \mathrm{HCW})$. Other complications were rash, shoulder pain, hand pain, cough, diarrhoea, nausea and vomiting in one $\mathrm{HCW}$ each. $14 \mathrm{HCW}$ in each group consumed paracetamol (dolo $650 /$ crocin/calpol/combiflam), two of them pre-emptively in Group-N, after first inoculation.

Adverse events recorded after second inoculation in Group-P were fever (5 $\mathrm{HCW}$ ), headache (5 HCW), myalgia (3 $\mathrm{HCW})$, malaise $(2 \mathrm{HCW})$ and pain at injection site $(4 \mathrm{HCW})$. One $\mathrm{HCW}$ reported anosmia. COVID-naïve $\mathrm{HCW}$ developed fever (5 HCW), headache (5 HCW), myalgia (3 HCW), malaise (2 $\mathrm{HCW})$ and pain at injection site $(4 \mathrm{HCW})$. Five $\mathrm{HCW}$ in Group-P (one of them prophylactically) and $2 \mathrm{HCW}$ in Group-N self-administered paracetamol after the second dose of vaccination.

\section{DISCUSSION}

A high $\operatorname{IgG}$ titre $(23.58 \pm 2.06 \mathrm{AU}$; min-max 19.7-27AU) was observed in all patients with previous exposure to SARS$\mathrm{CoV}-2$. And that too within 7-10 days of first dose of vaccination. This was irrespective of the initial antibody titre. The standard deviation in the COVID-survivors was much lower even at 1-2 weeks post first dose of vaccination. Vaccine prioritization needs to be practiced as vaccine is a limited resource. ${ }^{[3]}$ The goal of vaccination is to obtain protective levels of antibodies and if this purpose is met with a single dose of vaccine in COVID-survivors, then why not practice triage and vaccinate COVID-naïve individuals who are at greater risk of contracting COVID-19 by virtue of their immunity status, instead of inoculating COVID-survivors for the second time. Among COVID-naïve $\mathrm{HCW}$, three $\mathrm{HCW}$ had IgG titre <1AU (absence of protection), six HCW had a titre< 4.62AU corresponding to mild immunity, $14 \mathrm{HCW}$ had S/C values between 4.62 and 18AU corresponding to moderate levels of protection and remaining eight $\mathrm{HCW}$ had counts >18AU corresponding to high levels of protection. Only one study (a preprint) is available that reports quantitative IgG titres after the first dose of Covishield vaccination in Indian $\mathrm{HCW}^{[8]}$ They have reported a median of 61.5AU for the Covishield arm and 6AU for the Covaxin arm. They too have used CLIA technology similar to us but their reference range is wider (antibody level $<15 \mathrm{AU} / \mathrm{ml}$ are considered as seronegative or non-responders whereas as per our manufacturers brochure <1AU was the corresponding level. The corresponding lower and upper limit of their quantitative spike antibody kit is 3.8 and $400 \mathrm{AU} / \mathrm{ml}$ as compared to 0-70AU in our kit. Their interquartile range of $30-119.5$ is wide which corroborates with our large standard deviation for Covishield induced IgG S/C values in the COVID-naïve group.

The standard deviation in COVIDNaïve group (12.20 \pm 8.01$)$ was much higher, highlighting a much greater variation in response to first dose of vaccination as compared to COVID-survivors $(23.58 \pm$ 2.06AU). This type of variable immune response with a high standard deviation $(12.08 \pm 9.57)$ has been reported after COVID-19 infection in a cohort of 214 $\mathrm{HCW}$ belonging to a single institution. ${ }^{[9]}$ The mean IgG S/C values after first dose of vaccination in COVID-naïve group corroborate with mean values obtained after natural infection and so does the standard deviation when compared to results reported by Shah et al. ${ }^{[9]}$ This unpredictable response warrants either a blanket second dose of vaccination for all individuals (current scenario) or antibody testing before the second dose, so that the second dose maybe postponed till such time the titres fall below high levels of protection, thus prolonging the effective duration of protection provided by two successive doses of vaccine.

The IgG S/C counts obtained after first dose of vaccination in COVID survivors $(23.58 \pm 2.06 \mathrm{AU})$ were higher than IgG S/C counts obtained after second dose of vaccination in COVID-naïve $\mathrm{HCW}$ $(16.40 \pm 6.55 \mathrm{AU})$. The second inoculation 
did not lead to a clinically significant rise in IgG $\mathrm{S} / \mathrm{C}$ values in COVID-survivors $(25.48 \pm 2.79 \mathrm{AU})$. This implies that a second dose is not required in COVID-survivors with $\mathrm{IgG}$ S/C counts $>4.62 \mathrm{AU}$.

Six HCW had a count greater than $18 \mathrm{AU}$ (at 28-30 days after first dose of vaccination, but preceding the second dose) in COVID-naïve group. This could be due to several reasons. Firstly, these $\mathrm{HCW}$ might have contracted subclinical COVID19 infection which is quite possible, considering that a serosurvey done just before the vaccination drive in Delhi reported that $56 \%$ of the population had developed IgG antibodies to COVID-19. The first dose of vaccination in these six HCW might have served like a booster dose just like for HCW who are COVIDsurvivors (Group-P) and so they developed antibodies in the same range as Group-P. A second explanation could be that just like exposure to the natural infection produced IgG titres in all categories (mild, moderate high levels of protection) artificial exposure via inoculation too produces variable titres. This explanation is unlikely because, natural exposure/infection has a variable viral load (cycle-threshold/CT-value) in contrast to artificial exposure/infection in which vaccination deposits a fixed amount and known strain of virus into the deltoid muscle mass. But again, there is a possibility of variation in bioavailability of vaccine due to differences in muscle mass, muscle blood flow, and body weight of different vaccine-recipients. The immune response of different individuals to the same dose of vaccine may also be different due to inherent innate immunity. The HCW with a low titre might have developed a higher titre had more spacing been provided between the two doses. Since the GOI recommended that the second dose be taken at 28days, allowing a maximum spacing of 6weeks, only $\mathrm{IgG}$ titres at 28 days, maximum at 35 days post-first dose could be tested in our subset of patients. The COVID-naïve HCW were reluctant to wait beyond this timeperiod before getting the second/booster dose as they were apprehensive about contracting the infection during the long waiting period.

In vaccines with medium efficacy $(>50 \%)$, as per a mathematical model by Matiti et al, mortality rate can be halved by achieving only $35 \%$ optimal vaccination of the population. ${ }^{[10]}$ For a scenario with a combination of low vaccine efficacy and low vaccine-supply, vaccines should be given to the high-risk groups (eg elderly) first. The Indian picture is different because vaccine efficacy is high for Covishield (70$76 \% ; 100 \%$ efficacy in preventing severe/critical disease and hospitalization), ${ }^{[11,12]}$ covaxin ${ }^{[13]}$ (78-81\%) and Sputnik V [14] $(91.6 \%)$ and these are indigenously manufactured. When both vaccine efficacy and vaccination coverage/stocks are high, the vaccine prioritization switches to first vaccinating the high-transmission groups (younger adults and children). ${ }^{[10]} \mathrm{We}$ have a medium vaccine-supply and this can be augmented by redirecting the second dose from COVID-survivors to COVID-naïve individuals based on antibody -titre of COVID-survivors so that vaccination focus can shift to younger adults and children in accordance with the optimal vaccine allocation model. ${ }^{[10]}$

Four HCW in Group-P refrained from taking the second dose of vaccination as they equated their COVID-bout with the first dose of vaccination in COVID-naïve $\mathrm{HCW}$ and their first dose of vaccination to the second/booster dose recommended for COVID-naïve HCW. Hence, they believed that the second dose of vaccination for them was equitable to a third vaccine dose in COVID-naïve persons and would not further raise their IgG to more protective levels. Their existing high IgG titres would give them the required protection in conjunction with appropriate masks, social distancing and repeated hand-washing. The fact that none of the HCW in Group-P (including the ones who had taken just one jab) developed COVID-19 infection in the second wave strengthens this conjecture. Rate of re-infection worldwide is 
exceptionally low and only a handful of case-reports testify reinfection. [15] Neutralisation antibody titre is highly predictive of degree of immunological protection and the $50 \%$ protective neutralisation level was reported as $20 \%$ of the average convalescent level as per a study by Khoury et al. ${ }^{[16]}$ This supports our data on the basis of which we suggest omitting the second inoculation in convalescent individuals.

There were two distinct clusters in distribution of $\mathrm{IgG}$ count after first vaccination dose in HCW who developed COVID-19 despite vaccination. Either their counts were between 0.65 to $4.84 \mathrm{AU}$ (six $\mathrm{HCW}$ ) or between 13.4 to17.7 AU (four $\mathrm{HCW})$. In the first cluster, a low immunity maybe responsible but in the latter group the reason could be anything from antibody dependent enhancement to vaccine failure to overwhelming viral load.

The highest IgG S/C value obtained after both doses of vaccination was 31.3AU in our subjects. This is much lower than the highest IgG value obtained after natural infection with SARS-CoV-2 56.5AU. ${ }^{[9]}$

Mild adverse events amenable to over-the-counter analgesic-antipyretics ensued post vaccination. Those HCW with a pre-existing moderate/high antibody titre against SARS-CoV-2 displayed almost the same incidence of fever and local pain reaction at injection site on being inoculated as compared to HCW who were COVIDnaïve. In most cases pain at injection site developed 12 hours post inoculation. However, the incidence of chills, myalgia, headache, malaise and miscellaneous adverse events was $50-100 \%$ higher in COVID-survivors after their first inoculation. This implies that all $\mathrm{HCW}$ especially COVID-survivors should consume a paracetamol $650 \mathrm{mg}$ tablet prior to vaccination as pre-emptive analgesia and repeat it after 8 hours.

The incidence of adverse events was much lower following the second inoculation in both groups. There were no serious adverse events following immunization (AEFI) in our subjects although many like anaphylaxis, acute coronary syndrome, stroke, paraparesis, facial nerve palsy, cellulitis have been reported in literature. ${ }^{[11,12,17-19]}$ Until March 29, 2021, at least 617 serious AEFI had been reported pan-India, claiming 180 lives $(29.2 \%)^{[20]}$ leading to vaccine-hesitancy. Evidence from our study may allay the fears of general public regarding vaccine-related side-effects giving an impetus to the vaccination drive.

We selected the correct serological test (quantification of $\operatorname{IgG}$ antibody levels) that helps identify an adaptive immune response as against total antibody count (IgA; IgM; IgG) that merely detects current or past exposure to SARS-CoV-2. A major strength of our study on $64 \mathrm{HCW}$ is that it has addressed the clinically important research question of whether a second vaccine dose is required for COVIDsurvivors, and has produced clinically significant results.

The major limitation of this study is that only humoral immunity was analysed and not the T-cell immune response in addition, due to financial and logistic constraints.

\section{CONCLUSION}

All COVID-naïve persons must mandatorily be given both doses of vaccination since the IgG-titres reach protective levels only after the second inoculation. The second dose of vaccine provides no extra benefit in COVIDrecovered $\mathrm{HCW}$ since their $\mathrm{IgG}$ antibody levels cross the $18 \mathrm{AU}$ mark signifying high levels of protection after the first vaccination dose itself and triage of this resource to COVID-naïve population can ameliorate vaccine-shortage problems. Separate explicit guidelines for vaccination of corona-survivors should be formulated on a priority basis and further research with larger sample size is important. While both are protective, natural infection provides more robust immunity than vaccination. Pre-emptive paracetamol before vaccination 
may nullify any post-inoculation adverse effects even before they arise.

\section{ACKNOWLEDGEMENTS}

We deeply acknowledge with gratitude the help and support provided by Ms. Rekha Sharma (H.R. Department), Mr. Hemant (blood sample collection) and Dr Laxmi (Casualty) and all the HCW of RGCIRC who are COVID-survivors.

\section{Sources of Funding / Financial \\ Disclosures:}

Participants of the study were entitled to subsidy on IgG antibody testing by the institution.

\section{Conflict of Interest: None}

\section{Ethical Approval: Approved}

\section{REFERENCES}

1. Covishield vs Sputnik V vs Covaxin; know price, efficiency and side effects of 3 vaccines available in India. Available from https://english.jagran.com/india/covid19vaccines-covishield-vs-covaxin-vs-sputnikv-know-their-efficiency-cost-and-possibleside-effects-10026855. Last accessed 2021 June 4.

2. Cases across India. Available from https://www.mygov.in/covid-19. Last accessed 2021 June 4.

3. Bubar KM, Reinholt K, Kissler SM et al. Model-informed COVID-19 vaccine prioritization strategies by age and serostatus. Science. 2021 Jan 21.

4. Clem AS. Fundamentals of vaccine immunology. J Glob Infect Dis 2011;3(1): 73-78.

5. Theel ES, Harring J, Hilgart $\mathrm{H}$ et al. Performance Characteristics of Four High-Throughput Immunoassays for Detection of IgG Antibodies against SARSCoV-2. J Clin Microbiol. 2020;58(8).

6. VITROS Immunodiagnostic Products AntiSARS-CoV-2 IgG Reagent Pack 6199955. Available from https://www.fda.gov/media/137363/downlo ad. Last accessed 2021 Jun 5

7. von

Elm E, Altman DG, Egger M. Strengthening the reporting of observational studies in epidemiology (STROBE) statement: Guidelines for reporting observational studies. BMJ 2007;335:806-8.

8. Singh AK, Phatak SR, Singh NK, Gupta A, Sharma A, Bhattacharjee K. Antibody Response after First-dose of ChAdOx1nCOV (Covishield ${ }^{\mathrm{TM}^{\circledR}}$ ) and BBV-152 $\left(\right.$ Covaxin $^{\mathrm{TM}^{\circledR}}{ }^{\text {}}$ amongst Health Care Workers in India: Preliminary Results of Cross-sectional Coronavirus Vaccineinduced Antibody Titre (COVAT) study medRxiv 2021.04.07.21255078; doi: https:// doi.org/10.1101/2021.04.07.21255078

9. SB Shah, R Chawla, A Pahade et al. Immunity status of Health Care Workers post recovery from COVID-19: An online longitudinal panel survey. medRxiv 2020.11.27.20239426; doi: https:// doi.org/10.1101/2020.11.27.20239426

10. Khoury DS, Cromer D, Reynaldi A. What level of neutralising antibody protects from COVID-19? medRxiv preprint doi: https://doi.org/10.1101/2021.03.09.212 52641

11. Matrajt L, Eaton J, Leung T et al. Vaccine optimization for COVID-19: who to vaccinate first?. medRxiv. 2020 Jan 1.

12. Voysey M, Clemens SA, Madhi SA et al. Safety and efficacy of the ChAdOx $1 \mathrm{nCoV}$ 19 vaccine (AZD1222) against SARS-CoV2: an interim analysis of four randomised controlled trials in Brazil, South Africa, and the UK. The Lancet. 2021 Jan 9;397(10269):99-111.

13. Clinicaltrials.gov. A Phase III Randomized, Double-blind, Placebocontrolled Multicenter Study in Adults to Determine the Safety, Efficacy, and Immunogenicity of AZD1222, a Nonreplicating ChAdOx1 Vector Vaccine, for the Prevention of COVID-19. [Online] Available

at: https://clinicaltrials.gov/ct2/show/NCT 04516746 ?term $=$ NCT0 $4516746 \&$ draw $=2$ \&rank=1. Last accessed: February 2021.

14. Doroftei B, Ciobica A, Ilie OD et al. MiniReview Discussing the Reliability and Efficiency of COVID-19 Vaccines. Diagnostics. 2021 Apr;11(4):579.

15. Logunov DY, Dolzhikova IV, Shcheblyakov DV et al. Safety and efficacy of an rAd26 and rAd5 vector-based heterologous prime-boost COVID-19 vaccine: an interim analysis of a randomised 
controlled phase 3 trial in Russia. The Lancet. 2021 Feb 20;397(10275):671-81.

16. Duggan NM, Ludy SM, Shannon BC et al. Is novel coronavirus 2019 reinfection possible? Interpreting dynamic SARS-CoV2 test results through a case report. Am J Emerg Med 2020 Jul 4:S07356757(20)30583-

0. doi: 10.1016/j.ajem.2020.06.079. Epub ahead of print. PMID: 32703607; PMCID: PMC7335 242

17. Folegatti PM, Ewer KJ, Aley PK. Safety and immunogenicity of the ChAdOx1 nCoV-19 vaccine against SARS-CoV-2: a preliminary report of a phase $1 / 2$, singleblind, randomised controlled trial. Lancet 2020; 396: 467-78

18. Trogen B, Caplan A. Risk Compensation and COVID-19 Vaccines. Ann Int Med 2021; https://doi.org/10.7326/M20-
19. Oldenburg J, Klamroth R, Langer $\mathrm{F}$ et al. Diagnosis and Management of VaccineRelated Thrombosis following AstraZeneca COVID-19 Vaccination: Guidance Statement from the GTH. Hämostaseologie. 2021 Apr 1.

20. 617 Serious adverse events after vaccination reported in India until March 29. Available from

https://science.thewire.in/health/617serious-adverse-events-after-vaccinationreported-in-india-until-march-29/. Last accessed 2021 Jun 5.

How to cite this article: Shah SB, Chawla R, Mehta A et.al. SARS-CoV-2: comparison of active immunity acquired by natural exposure versus inoculation of HCW. Gal Int J Health Sci Res. 2021; 6(2): 54-63. DOI: https://doi.org/10. 52403/gijhsr.20210408 